Original Paper http://ajol.info/index.php/ijbcs http://indexmedicus.afro.who.int

\title{
Distribution spatiale et saisonnière des gîtes larvaires des moustiques dans les espaces agricoles de la zone de Mouila, Gabon
}

\author{
Aubin Armel KOUMBA ${ }^{1,2^{*}}$, Christophe Roland ZINGA KOUMBA ${ }^{1}$, \\ Rodrigue MINTSA NGUEMA ${ }^{1}$, Luc Salako DJOGBENOU ${ }^{2,3}$, Pyazzi OBAME ONDO ${ }^{4}$, \\ Guillaume Koffivi KETOH ${ }^{5}$, Pearl $\mathrm{COMLAN}^{6}$, Bertrand $\mathrm{M}^{\text {'BATCHI }}{ }^{7}$ et \\ Jacques François MAVOUNGOU ${ }^{1,7}$ \\ ${ }^{1}$ Institut de Recherche en Ecologie Tropicale (IRET), BP : 13354, Libreville, Gabon. \\ ${ }^{2}$ Université d'Abomey-Calavi (UAC), 05 BP : 1604, Cotonou, Benin. \\ ${ }^{3}$ Institut Régional de Santé Publique (IRSP), BP : 918, Ouidah, Bénin. \\ ${ }^{4}$ Programme National de Lutte contre le Paludisme (PNLP), BP : 14426, Libreville, Gabon. \\ ${ }^{5}$ Unité de Recherche en Ecotoxicologie, Université de Lomé (UL), BP : 1515, Lomé, Togo. \\ ${ }^{6}$ Université des Sciences de la Santé (USS), BP : 4009, Libreville, Gabon. \\ ${ }^{7}$ Université des Sciences et Techniques de Masuku (USTM), BP : 941, Franceville, Gabon. \\ *Auteur correspondant ; E-mail : aubinho25@yahoo.fr; Tél: 0024106661001.
}

\section{RESUME}

Les moustiques sont des sources de nuisances et des vecteurs d'agents pathogènes pour l'homme et les animaux. La lutte contre ces insectes nécessite la connaissance de leurs lieux de développement. C'est dans ce contexte qu'une étude sur la connaissance de la distribution spatio-temporelle des gîtes des moustiques a été conduite à Mouila, au Sud-Ouest du Gabon, dans les zones d'exploitation de palmier à huile. Ainsi, des prospections des gîtes larvaires ont été effectuées en saison sèche et en saison pluvieuse et, les paramètres physico-chimiques de ces habitats ont été mesurés. Au total, 178 gîtes larvaires ont été identifiés et repartis en 47 contenants artificiels, 9 surfaces d'eau naturelles et 122 surfaces d'eau artificielles. Les surfaces d'eau artificielles ont été plus abondantes que les contenants artificiels et les surfaces d'eau naturelles. La majorité des gîtes larvaires se trouvait dans l'environnement immédiat des populations humaines $(<400 \mathrm{~m})$. Le maximum de gîtes larvaires a été obtenu en saison des pluies $(68,54 \%)$ et le minimum en saison sèche (31,46\%). Au total, 12136 larves de moustiques ont été récoltées dont 2926 étaient des larves d'Anopheles gambiae s.l. et 9210 étaient des larves de Culex sp et d'Aedes sp. Par ailleurs, sur 6329 imagos émergés, 5173 moustiques adultes ont été récoltés à la saison des pluies et 1156 moustiques adultes à la saison sèche. Cette étude indique que les moustiques se développent dans tous les types de points d'eau mais préfèrent les surfaces d'eau artificielles. La pullulation de ces moustiques serait liée à la pluviométrie, à l'insalubrité de l'environnement immédiat et aux activités anthropiques créant des sites de reproduction des moustiques.

(C) 2018 International Formulae Group. All rights reserved.

Mots clés : Moustiques, gîtes larvaires, vecteurs, maladies, Mouila, Gabon. 


\title{
Seasonal and spatial distribution of breeding sites of mosquitoes in the agricultural spaces in the Mouila area, Gabon
}

\begin{abstract}
The mosquitoes are a source of nuisance and vectors of pathogens for humans and animals. The control of these vectors requires knowledge of their breeding sites. This study focuses on knowledge of the spatial and temporal distribution of mosquito larval habitats in Mouila (south-west of Gabon). Larval surveys in various water collections and the measurement of physic and chemical parameters were carried out in the oil palm exploitation sites, in the Mouila area, in August 2017 (dry season) and in October 2017 (rainy season). 178 breeding sites were identified and divided into 47 artificial containers, 9 natural water surfaces and 122 artificial water surfaces. The artificial water surfaces were more abundant than artificial containers and natural water surfaces. The majority of positive larval habitats $(94.81 \%)$ were in the immediate environment of human populations $(<400 \mathrm{~m})$. The maximum of breeding sites was obtained in the rainy season $(68.54 \%)$ and the minimum in the dry season (31.46\%). Moreover, 12136 mosquito larvae were collected, of which 2926 were Anopheles gambiae s.l. larvae and 9210 were Culex spp and Aedes spp. larvae. On overall 6329 adult mosquitoes obtained, 5173 adult mosquitoes were collected in the rainy season and 1156 adult mosquitoes in the dry season. This study indicates that mosquitoes grow in all types of water collections but they prefer the artificial water surfaces. The proliferation of these mosquitoes would be linked to pluviometry, insalubrity of immediate environment, human activities creating mosquito breeding sites.
\end{abstract}

(C) 2018 International Formulae Group. All rights reserved.

Keywords: Mosquitoes, larval breeding sites, vectors, diseases, Mouila, Gabon.

\section{INTRODUCTION}

Les moustiques constituent un problème majeur en santé humaine. En effet, ils sont impliqués dans la transmission de plusieurs agents pathogènes responsables de maladies telles que le paludisme, le chikungunya, les filarioses, les encéphalites, la fièvre de la vallée du Rift, la fièvre jaune, la fièvre Zika (Ikram et al., 2016). Ces maladies à transmission vectorielle figurent parmi les plusimportantes en santé humaine et animale (Becker et al., 2010). Par ailleurs, ces insectes sont de sérieuses sources de nuisances pour l'homme et les animaux (Serandour, 2007).

$\mathrm{Au}$ Gabon, plusieurs espèces de moustiques vecteurs d'agents pathogènes sont présentes (Paupy et al., 2013). Ces espèces de moustiques exploitent presque tous les types de collectionsd'eau pour pondre leurs œufs (Mourou et al., 2012). Par ailleurs, en raison de sa position par rapport à l'équateur, des facteurs climatiques et de la diversité des écosystèmes, le Gabon recèle de nombreuses régions favorables au développement des insectes hématophages (Mounioko et al., 2015 ; Mounioko et al., 2018). De plus, il estl'un des pays d'Afrique subsaharienne touché par le paludisme, le chikungunya, la dengue, le virus zika, la fièvre jaune (Elissa et al., 2003 ; Grard et al., 2014).

Actuellement, les moustiques, vecteurs de ces maladies, évoluent dans des systèmes écologiques naturels et ceux modifiés par l'homme (Makanga, 2016). En effet, le développement des plantations de palmier à huile au Gabon, particulièrement dans la région de Mouila, a entraîné une modification des paysages naturels et la mise en eau des palmeraies (Ndjimbi, 2013). Ces nouvelles conditions, favorables au développement larvaire et à la prolifération des moustiques, ont engendré une forte augmentation des cas de paludisme dans cette région (Koumba et al., 2018a ; 2018b).

Pour faire face à cette problématique des moustiques vecteurs d'agents pathogènes, l'Organisation Mondiale de la Santé (OMS) préconisela mise en place dela lutte anti vectorielle axée sur l'assainissement du cadre de vie, la distribution des Moustiquaires Imprégnées d'Insecticide à Longue Duréed'Action (MILDA) et la Pulvérisation 
Intra Domiciliaire d'insecticide à effet rémanent (PNLP, 2010 ; Badolo et al., 2012 ; Abagli et al., 2014). Mais, ces interventions nécessitent une meilleure connaissance de la bio écologie des vecteurs. Or, les informations sur les moustiques de la zone de Mouila demeurent encore insuffisantes. De plus, ces connaissances sont essentielles pour mieux orienter les actions de lutte (Koudou et al., 2005 ; Diedhiou et al., 2016). L'objectif de cette étude était de déterminer la distribution spatiale et saisonnière des gîtes larvaires des moustiques dans les sites d'exploitation de palmier à huile et leurs environs à Mouila.

\section{MATERIEL ET METHODES \\ Zone d'étude}

Les prospections et collectes larvaires ont été réaliséesdans la périphérie de la ville de Mouila (Sud-Ouest du Gabon), précisément dans les sites d'exploitation de palmier à huile (Mboukou, Moutassou) et dans certains villages environnants (Figure 1). La région de Mouila est située sur le $2^{\text {ème }}$ degré de latitude Sud. Elle est soumise à un climat de type équatorial de transition de la zone centrale du Gabon, avec une saison sèche de 3 mois (juillet à septembre) et une longue saison pluvieuse de 9 mois marquée par des orages fréquents et des pluies abondantes (octobre à juin). Avec le dérèglement climatique, il n'est pas rare que des rares pluies viennent perturber la saison sèche (150 mm par mois). Le maximum de pluies étant enregistré en octobre, novembre et mars $(400 \mathrm{~mm})$. La région reçoit entre 1800 et $2000 \mathrm{~mm}$ d'eau/an. Le taux d'humidité relative est aux alentours des $80 \%$ tandis que les températures moyennes mensuelles sont très régulières et oscillent entre $25^{\circ} \mathrm{C}$ et $32{ }^{\circ} \mathrm{C}$ (Ecosphère, 2011).

$\mathrm{Au}$ plan topographique, la région de Mouila est formée de nombreuses dépressions séparées par des collines aux flancs plus ou moins raides. Globalement, on retrouve dans cette région les sols ferralitiques (partout où le drainage est bon), les sols hydromorphes (dans les collines et les axes de drainage) et les sols peu évolués (au niveau des pentes ou le sommet des buttes) (Ecosphère, 2011; 2014). Sur le plan géomorphologique, les couches géologiques de la région de Mouila s'inscrivent dans l'histoire géologique du Gabon. En effet, cette zone est située sur le socle archéen constitué d'un ensemble cristallin et cristallophylien. Il est formé du massif nord-Gabon, le massif du Chaillu et la chaine du Mayombe qui sont les éléments qui apparaissent en antéclise du craton congolais. Le dernier métamorphisme subi par ce socle a été daté de 2700 millions d'années. Ce vieux socle d'âge archéen, formé de gneiss granitisés (migmatites) et de granites souvent orthogneissifiés, est divisé en trois unités qui tendent à être individualisé. Le réseau hydrographique y est dominé par la présence de la Ngounié, rivière importante qui sert d'axe d'écoulement et de divers cours d'eau secondaires (Ecosphère, 2011 ; 2014). Le site de Mboukou ou «lot $1 »$ est une zone de palmeraies qui se trouve dans le département de Tsamba-Magotsi à environ 35 kilomètres de la ville de Mouila. Ce site est limité à l'Est par la rivière Ngounié et les villages SaintMartin et Migabe puis à l'Ouest par les villages Douya, Doubou, Mboukou et Rembo (Ecosphère, 2011). Ce site industriel s'étend sur près 35300 hectares et se trouve entre $1^{\circ} 39^{\prime} 06^{\prime \prime}$ de latitude Sud et 1049'42,6" de longitude Est (Ecosphère, 2011).Il est drainé par la rivière Ngounié, qui est le principal cours d'eau et, des rivières secondaires comme Douya, Doubou, Rembo et Igege (Ecosphère, 2011).Quant au site de Moutassou ou «lot $3 »$, il est la troisième zone de plantation de palmiers à huile. Ce site agroindustriel couvre 23780 hectares et se trouve dans le département de la Douya-Onoye à environ 13 kilomètres de la ville de Mouila (Ecosphère, 2014). Il est situé entre 1'59'33,8" de latitude Sud et $11^{\circ} 02^{\prime} 25,2^{\prime \prime}$ de longitude Est. Il est limité au Nord par la ville de Mouila, à l'Est par les villages Moulandoufouala, Mbengui et Mbadi puis à l'Ouest par les villages Moutassou, Koumbanou, Ikolo-Ikolo, Digabosse. C'est un 
écosystème composé de trois grands bassins hydrographiques que sont le bassin de la Douya et ses quatre affluents (Koutsou, Monfi , Doukoutsou et Gandji), le bassin de Gidighi avec ses deux petites rivières (Idighi et Baghamba) et le bassin Dibotsa avec ces trois affluents que sont Grand Mbatsi, Petit Mbatsi et Dwiba (Ecosphère, 2014). Il est marqué par des mosaïques de forêt-savane dominées par les savanes qui occupent environ $75 \%$ du permis d'exploitation (Ecosphère, 2014).

\section{Prospection et identification des gîtes larvaires}

Les prospections des gîtes larvaires ont été faites dans 9 stations de collecte sélectionnées dans la zone d'étude. Ces stations ont été représentées par: le Pk19, le camp Moutassou (Estate 13, Estate 13Bis, Estate 15), le village Moutambe Sane Foumou, le camp Doubou, le village Mboukou, le village Doubou, le camp Mavassa, le camp Ngounié, le camp Mboukou. Le choix porté sur ces stations a été orienté par leur profil écologique et lastructuration du paysage. Ces prospections ont été réalisées en août 2017 (saison sèche) et en octobre 2017 (saison des pluies). Les prospections ont consisté à rechercher systématiquement tous les gîtes larvaires potentiels de moustiques, à les identifier et à effectuer des prélèvements de larves dans les gîtes positifs (Tia et al., 2016). Ainsi, à chaque passage dans une station, les collections d'eau susceptibles d'héberger des stades pré-imaginaux de moustiques ont été prospectées (Coffinet et al., 2009).

La prospection des gîtes comprenait d'abord l'observation directe de la présence ou non de larves de moustiques (Sy et al., 2016). En cas de présence de ces stades immatures, des prélèvements des larves et des nymphes étaient effectués par la méthode de dipping (Coffinet et al., 2009 ; Talipouo et al., 2017) et au moyen d'une louche de $300 \mathrm{~mL}$. Cette méthode à consister à réaliser une dizaine de plongées de louche à plusieurs endroits du point de récolte afin de maximiser les captures (Talipouo et al., 2017). Ainsi, les stades pré-imaginaux des moustiquescapturés étaienttransférés dans des plateaux à l'aide des pipettes de transfert puis transportés dans des glacières au laboratoire. Les différentes larves récoltées étaient ensuite triées et dénombrées par sous-famille (Anophelinae ou Culicinae). Ces larves et nymphes ont été élevées jusqu'à l'obtention des moustiques adultes (Koné et al., 2013; Tia et al., 2016). Ilsont été identifiés morphologiquement au niveau du genre (Anopheles, Culex, Aedes) sous une loupe binoculaire (Akono et al., 2017), à l'aide de la clé de détermination de Baldacchino et Paupy (2010) et le logiciel de détermination des Culicidae de l'ORSTOM.

Parallèlement, divers paramètres des gîtes larvaires de moustiques ont été relevésin situà l'aide du GPSMAP 62s de marque Garmin, dumulti paramètre portatif HANNA HI 9828et du mètre (Sy et al., 2016). Les informations suivantes ont été prises : le type de collection d'eau, l'origine de l'eau, la présence de larves ou de nymphes de moustiques, les coordonnées géographiques, la taille et la profondeur du gîte, la température et le $\mathrm{pH}$ de l'eau du gîte(Kenawy et al., 2013).

\section{Analyse des données}

Les données collectées ont été saisies dans Excel office professionnel version 2013 et analysées avec le logiciel $\mathrm{R}$ version 3.2.2. Les pourcentages et les effectifs ont été calculés dans le tableur Excel. Le test de Khi 2 de Pearson a été utilisé pour comparer les proportions des larves dans les différents gîtes et entre les saisons. Le seuil de significativité de ces testsétait de 5\%. Par ailleurs, les données sur les gîtes larvaires et les habitations humaines ont été importées dans le logiciel de cartographie ArcGIS version 10.1 pour l'élaboration des cartes et la réalisation des analyses spatiales. 


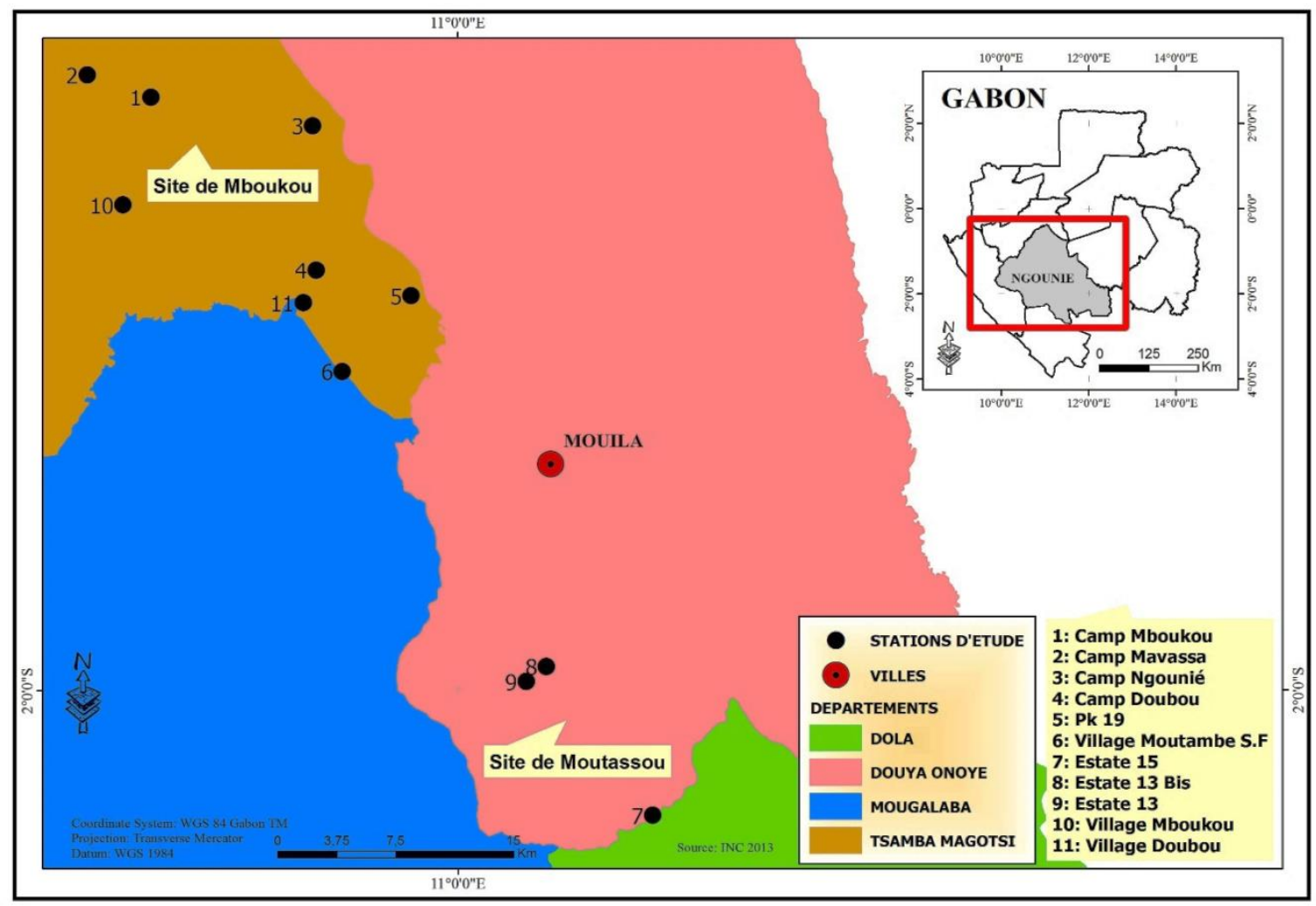

Figure 1 : Localisation géographique des sites d'étude et des stations prospectées.

\section{RESULTATS \\ Caractérisation quantitative et qualitative des gîtes larvaires}

Au total, 178 gîtes larvaires ont été identifiés au cours de cette étude. Ces gîtes ont été répartis en 47 contenants artificiels (boîtes vides, des tonneaux, fûts, etc.), 9 surfaces d'eau naturelles (rivières, étangs, etc.) et 122 surfaces d'eau artificielles (flaques d'eau temporaire issues des ménages et autres activités anthropiques). Dans cette zone d'étude, on a dénombré plus de surfaces d'eau artificielles que de surfaces d'eau naturelles. Cependant, il n'existe pas de différence significative dans la répartition des gîtes en fonction du type de gîte larvaire $\left(\mathrm{X}^{2}=3,636\right.$; $\mathrm{df}=2 ; \mathrm{p}=0,162)$. Par ailleurs, le maximum de gîtes larvaires a été observé en saison des pluies (122 gîtes, soit 68,54\%) et le minimum en saison sèche (56 gîtes, soit $31,46 \%)$. Toutefois, il n'y a pas de différence significative dans la distribution de ces gîtes larvaires en fonction de la saison $\left(\mathrm{X}^{2}=3,63\right.$; $\mathrm{p}=0,14)$.
Au total, 106 (86,88\%) gîtes larvaires étaient temporaires et 16 autres gîtes étaient permanents soit $13,12 \%$ en saison des pluies. Par contre, en saison sèche, 31 gîtesétaient temporaires $(55,36 \%)$ et 25 gîtes étaient permanents $(44,64 \%)$ (Figure 2).

Variation saisonnière de la positivité des gites larvaires de moustiques

Parmi les 178 gîtes larvaires identifiés dans la zone d'étude, 117 gîtes $(65,73 \%)$ étaient positifs c'est-à-dire contenaient au moins une larve de moustique, alors que 61 autres gîtes $(34,27 \%)$ étaient négatifs. De même, il existe une différence significative dans la distribution des larves dans les gîtes larvaires $\left(\mathrm{X}^{2}=1,178 ; \mathrm{p}=0,278\right)$. Par ailleurs, 77 gîtes positifs $(63,11 \%)$ et 45 gîtes négatifs $(36,89 \%)$ ont été identifiés en saison des pluies dans les stations prospectées, contre 40 gîtes positifs $(71,43 \%)$ et 16 gîtes négatifs $(28,57 \%)$ saison sèche (Figure 3$)$.

Par ailleurs, parmi les 77 gîtes positifs identifiés en saison pluvieuse, 23 gîtes 
(29,87\%) abritaient des larves d'Anophelinae (Anopheles gambiae s.l.), 38 gîtes $(49,35 \%)$ hébergeaient des larves de Culicinae (Culex $s p$. et Aedes sp.) et 16 gîtes (20,78\%) étaient mixtes (contenaient à la fois des larves d'Anophelinae et celles de Culicinae). Cependant en saison sèche, les 40 gîtes positifs répertoriés étaient composés de 3 gîtes à Anophelinae (7,5\%), 27 gîtes à Culicinae $(67,5 \%)$ et 10 gîtes mixtes $(25 \%)$. Le tableau 1 récapitule les caractéristiques physicochimiques des gîtes positifs rencontrés dans la zone d'étude. Les larves d'An. Gambiae s.l. (Anophelinae) ont été rencontrées dans des gîtes à $\mathrm{pH}$ soit acide ou neutre $(6,77 \pm 0,24)$ et à température légèrement plus faible $(26,68 \pm$ $3,57{ }^{\circ} \mathrm{C}$ ) que celle des gîtes de Culicinae $\left(28,38 \pm 3,87^{\circ} \mathrm{C}\right)$.

\section{Pourcentage des larves récoltées dans la zone d'étude}

Dans la zone d'étude, 12136 larves de moustiquesont été récoltées dont 2926 $(24,11 \%)$ de la sous-famille des Anophelinae et $9210 \quad(75,89 \%)$ de la sous-famille des Culicinae (Figure 5). Par ailleurs, 7867 $(64,82 \%)$ larves ont été récoltées en saison des pluies contre $4269(35,18 \%)$ en saison sèche $\left(\mathrm{X}^{2}=478,57 ; \mathrm{p}=2\right.$, 2e-16). Parmi les 12136 larves de moustiques récoltées dans la zone d'étude, le maximum de larves a été obtenu au village Moutambe Sane Foumou $(n=3282$; 27,04\%), dans les camps Moutassou $(\mathrm{n}=2907 ; 23,95 \%), \quad$ Mboukou $(\mathrm{n}=1945$; $16,03 \%)$ et Ngounié $(\mathrm{n}=1304 ; 10,74 \%)$. De même, le camp Moutassou (1514 larves), le village Moutambe Sane Foumou (1162 larves) et le camp Mboukou (929 larves) ont été les stations les plus productives en saison sèche (Tableau 4). Or, en saison des pluies, la production larvaire a été maximale au village Moutambe Sane Foumou (2120 larves), au camp Moutassou (1393 larves), au camp Ngounié (1121 larves) et au camp Mboukou (1016 larves) (Tableau 2).

Variation de l'abondance des larves dans les gîtes en fonction des saisons et des stations

Dans la zone d'étude, l'abondance des larves au niveau des gîtes varie en fonction des stations prospectées et de la saison. En effet, au cours de la saison sèche, plusieurs stations ont été très productives en larves de Culicinae. Au total, 1330 larves de cette sousfamille ont été échantillonnées dans le camp Moutassou, 1073 dans le village Moutambe Sane Foumou et 685 dans le camp Mboukou, soit des pourcentages respectifs de 35,6\%; $28,8 \%$ et $18,4 \%$. Quant aux Anophelinae, leurs larves ont été récoltées majoritairement au camp Moutassou, camp Mboukou, camp Ngounié et au village Moutambe Sane Foumou (Tableau 3). Ainsi, 184 larves ont été collectées dans le camp Moutassou (34,3\%) et 244 dans le camp Mboukou (45,4\%).

En saison des pluies, les gîtes du village Moutambe Sane Foumou $(\mathrm{n}=2114$; $38,59 \%)$, du camp Ngounié $(\mathrm{n}=1037$; $18,93 \%)$, du camp Mboukou ( $\mathrm{n}=765$; $13,96 \%)$ et du camp Moutassou ( $n=614$; $11,21 \%)$ ont été les plus fructueux en larves de Culicinae (Tableau 3). Pour la sous-famille des Anophelinae, ce sont les gîtes du camp Moutassou ( $\mathrm{n}=779 ; 32,61 \%)$, du village Mboukou $(\mathrm{n}=700 ; 29,30 \%)$ et du camp Mavassa ( $\mathrm{n}=518 ; 21,68 \%)$ qui ont été les plus productifs de tous (Tableau 3 ).

Identification des adultes des sous familles des moustiques par saison et par station

Un total de 6329 moustiques adultes dont $5173(81,73 \%)$ en saison des pluies contre $1156(18,27 \%)$ en saison sèchea été obtenu à l'issue del'élevage des larves prélevées au cours des prospections. Aussi, il existe une différence significative des adultes de moustiques obtenus en fonction des saisons $\left(\mathrm{X}^{2}=32,54 ; \mathrm{df}=1 ; \mathrm{p}=1,168 \mathrm{e}-08\right)$.Parmi ces imagos, il y avait plus d'adultes de la sousfamille des Culicinae (3663 imagos en saison des pluies et 916 en saison sèche) que ceux de la sous-famille des Anophelinae (1510 adultes en saison des pluies et 240 en saison sèche) (Tableau 4) sèche $\left(\mathrm{X}^{2}=32,54 ; \mathrm{df}=1 ; \mathrm{p}=\right.$ $1,168 \mathrm{e}-08)$. Ces moustiques adultes se répartissaient en trois genres, à savoir Anopheles, Culex et Aedes.

Le tableau 5montre la répartition des moustiques adultes ayant émergé en fonction des stations prospectées dans la région de Mouila. Cette répartition des imagos varie en fonction des saisons. En effet, en saison sèche, les adultes de moustiques ont été 
majoritairement enregistrés au camp Mboukou $(n=639 ; 55,28 \%)$ et au camp Moutassou ( $n=294 ; 25,43 \%)$. En revanche, en saison pluvieuse, les moustiques adultes ont été plus abondants au village Moutambe Sane Foumou $\quad(n=1702 ; 32,90 \%)$, au camp Mboukou $(\mathrm{n}=910 ; 17,59 \%)$, au camp Moutassou $(\mathrm{n}=842 ; 16,28 \%)$, au camp Ngounié $(n=604 ; 11,68 \%)$ et au camp Mavassa ( $\mathrm{n}=509 ; 9,84 \%)$.

Quant à la distribution saisonnière de ces adultes, on a constaté que les stations les plus productives en adultes d'Anophelinae (Anopheles gambiae s.l.) pendant la saison sèche ont été le camp Mboukou (170 adultes, soit 70,83\%), le camp Moutassou (46 imagos, soit $19,17 \%$ ) et le village Moutambe Sane Foumou (13 adultes, soit 5,42\%). Par contre, les gîtes larvaires les plus fructueux en adultes de Culicinae (Culex sp. et Aedes sp.) au cours de la saison sèche ont été ceux du camp Mboukou $(n=469 ; 51,20 \%)$ et du camp Moutassou $(n=248 ; 27,07 \%)$. Les autres stations ont présenté des effectifs de moustiques adultes inférieurs ou égaux à 52 adultes $(5,12 \%)$ (Tableau 5).

De même, en saison pluvieuse, la répartition des moustiques adultes de ces deux sous-familles était variable en fonction des stations prospectées. En effet, les adultes d'Anophelinae (Anopheles gambiae s.l.) obtenus après élevage pendant cette saison, provenaient essentiellement de quatre stations dont le camp Moutassou ( $\mathrm{n}=524 ; 34,70 \%)$, le camp Mavassa $(n=311 ; 20,60 \%)$, le village Mboukou $(n=306 ; 20,26 \%)$ et le camp Mboukou $(n=251 ; 16,62 \%)$. Les autres stations ont présenté moins de $8 \%$ des adultes obtenus après élevage (Tableau 5).A l'opposé, les gîtes larvaires les plus productifs en adultes de Culicinae (Culex sp. et Aedes sp.) au cours de la saison sèche ont été ceux du village Moutambe Sane Foumou (1696 adultes, soit 46,30\%), du camp Mboukou (659 adultes, soit 17,99\%) et du camp Ngounié (527 imagos, soit 14,39\%). Ces trois stations d'étude ont produit $2882 \quad(78,68 \%)$ moustiques adultes de Culicinae. Les autres stations prospectées, notamment le camp Moutassou, le Pk 19, le camp Mavassa, le village Doubou, le camp Doubou et le village Mboukou ont présenté des effectifs de moustiques adultes respectifs de $318(8,68 \%)$; $220(6,01 \%) ; 198(5,41 \%) ; 33(0,90 \%) ; 12$ $(0,33 \%)$ et $0(0 \%)$.

\section{Localisation des habitats larvaires et leur proximité aux habitations}

La localisation des gîtes larvaires de moustiques varie en fonction des stations prospectées. En effet, le maximum de gîtes positifs était identifié dans le camp Moutassou $(n=33 ; 28,20 \%)$ et dans le village Mboukou $(\mathrm{n}=15 ; 12,82 \%)$, suivi du village Moutambe Sane Foumou $(n=12 ; 10,26 \%)$, du camp Mboukou ( $n=12 ; 10,26 \%)$, du camp Ngounié $(\mathrm{n}=12 ; 10,26 \%)$ et du camp Mavassa $(\mathrm{n}=11$; 9,4\%) (Tableau 5).

De plus, ces effectifs ont varié selon la saison. Par exemple, en saison sèche, le maximum de gîtes positifs collecté a été obtenu dans le camp Moutassou ( $n=13$; $32,5 \%)$ et dans le village Moutambe Sane Foumou ( $\mathrm{n}=8 ; 20 \%)$, suivi du camp Mboukou $(\mathrm{n}=5 ; 12,5 \%)$ et du camp Doubou $(\mathrm{n}=5$; $12,5 \%$ ) (Tableau 6). Par contre, pendant la saison pluvieuse, le maximum de gîtes positifs a été répertorié dans le camp Moutassou $(25,97 \%)$, le village Mboukou $(16,88 \%)$ et dans le camp Mavassa (12,99\%).

La figure 6 présente la variation des gîtes larvaires positifs des moustiques selon la distance aux habitations humaines. Seules les données de la saison des pluies ont été représentées car on a noté une forte abondance des gîtes positifs durant cette période contrairement à la saison sèche. En effet, la majorité des gîtes positifs était située entre 0 et $100 \mathrm{~m}$ des habitations humaines. Ces gîtes représentaient $75,32 \%$ de tous les gîtes positifs identifiés $(n=58 / 77)$. Le pourcentage de gîtes positifs a diminué au fur et à mesure que l'on s'éloignait des maisons. Ainsi, $75,32 \%$ des gîtespositifs ont été localisés à moins de $100 \mathrm{~m}$ des habitations. Cependant, à plus de 1000-2000 $\mathrm{m}$ de distance de ces habitations, seulement $5,19 \%$ des gîtes positifs ont été recensés. Globalement, à moins de 400 $\mathrm{m}$ des habitations, on a identifié près de $94,81 \%(n=73 / 77)$ des gîtes positifs. 


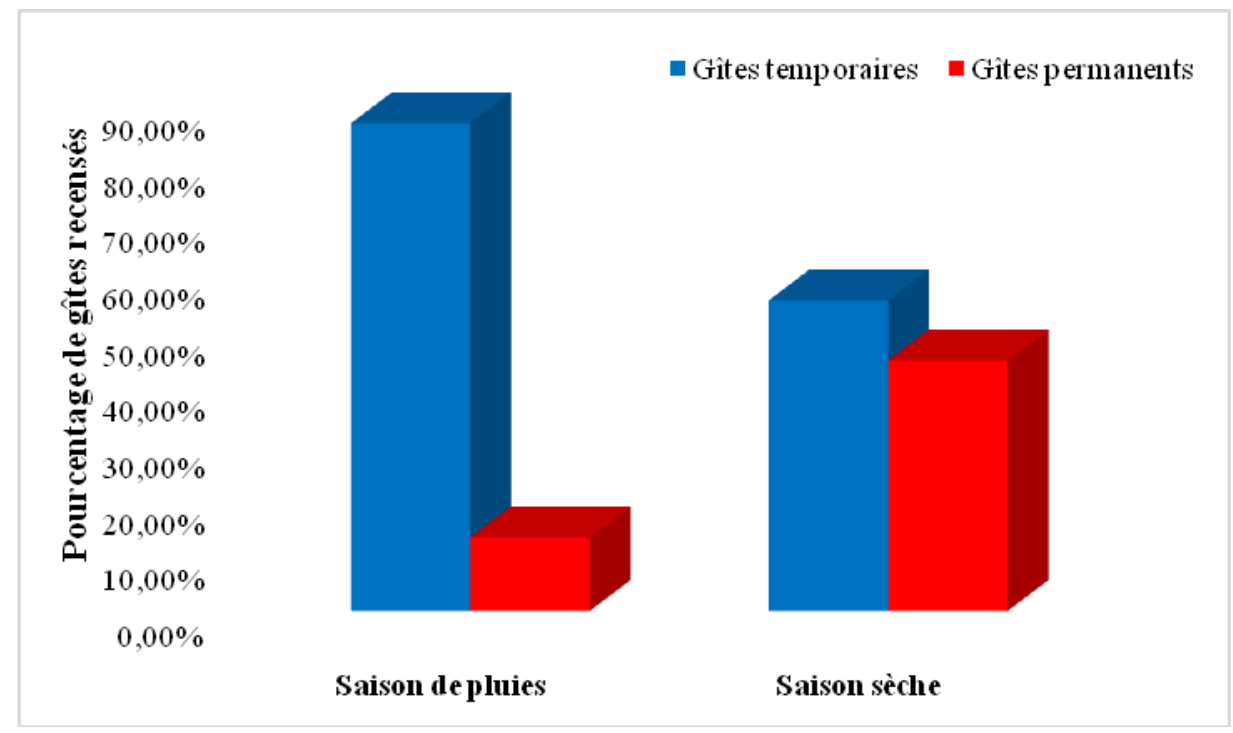

Figure 2 : Variation saisonnière des gîtes temporaires et permanents.

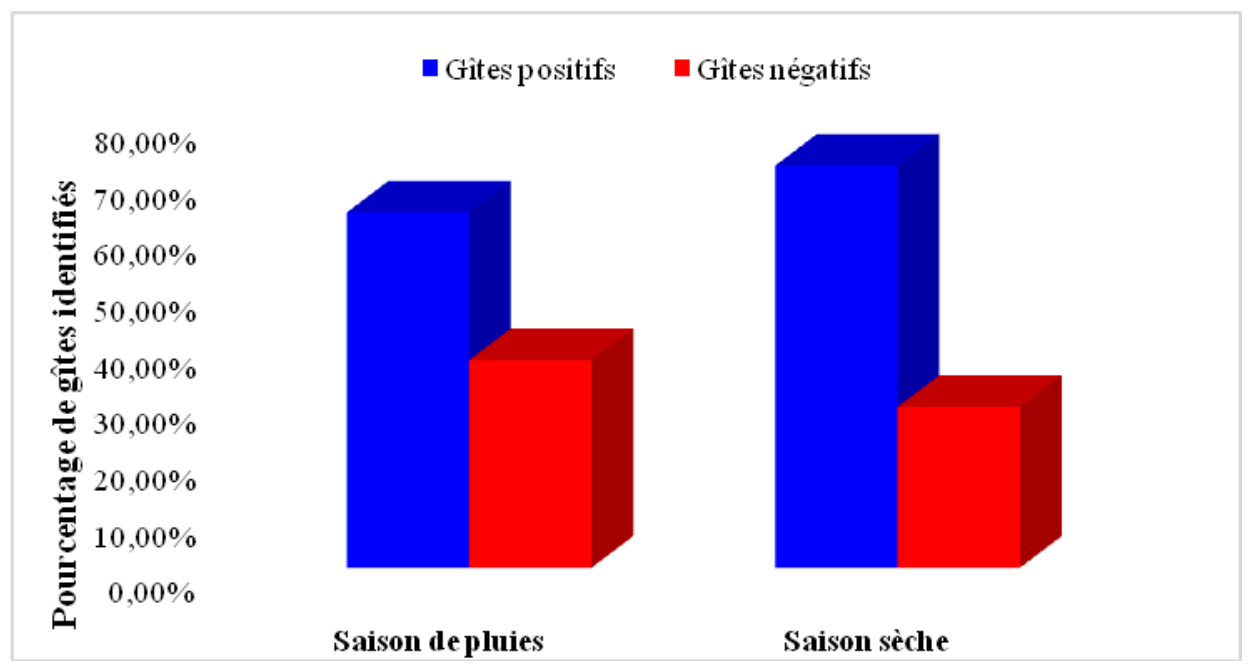

Figure 3 : Répartition de la positivité des gîtes identifiés en fonction des saisons.

Tableau 1 : Caractéristiques physico-chimiques des gîtes larvaires positifs de moustiques.

\begin{tabular}{clllc}
\hline & & & & \\
Température $\left({ }^{\circ} \mathbf{C}\right)$ & Profondeur $(\mathbf{m})$ & Surface $\left(\mathbf{m}^{2}\right)$ & $\mathbf{p H}$ \\
\hline GA & $26,68 \pm 3,57$ & $0,28 \pm 0,23$ & $11,50 \pm 8,50$ & $6,77 \pm 0,24$ \\
GM & $27,4 \pm 3,84$ & $0,27 \pm 0,24$ & $500,02 \pm 499,98$ & $6,65 \pm 0,19$ \\
GC & $28,38 \pm 3,87$ & $0,56 \pm 0,55$ & $60,03 \pm 59,97$ & $7,35 \pm 0,95$ \\
\hline
\end{tabular}




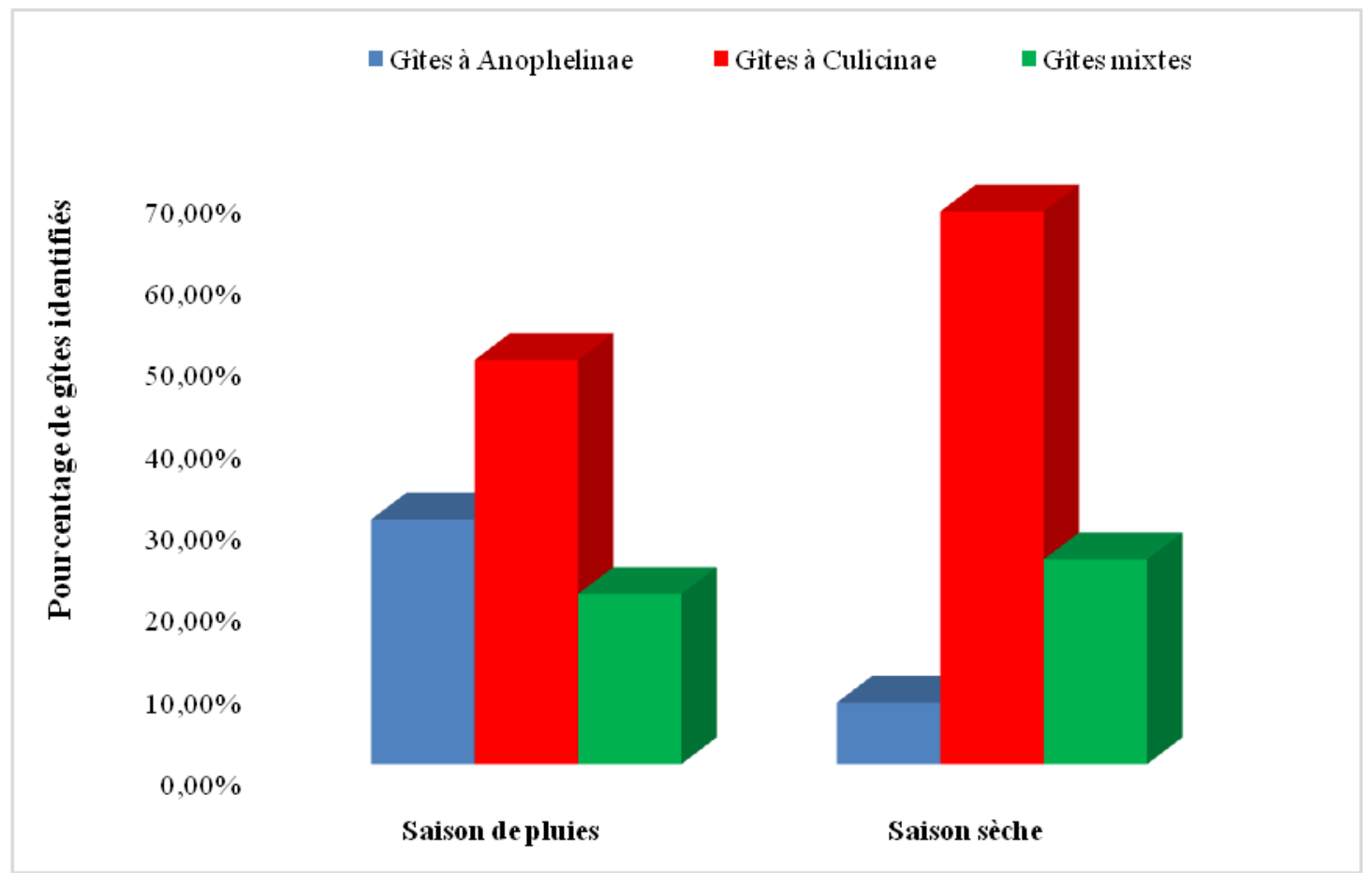

Figure 4 : Répartition saisonnière des types de gîtes positifs à Mouila.

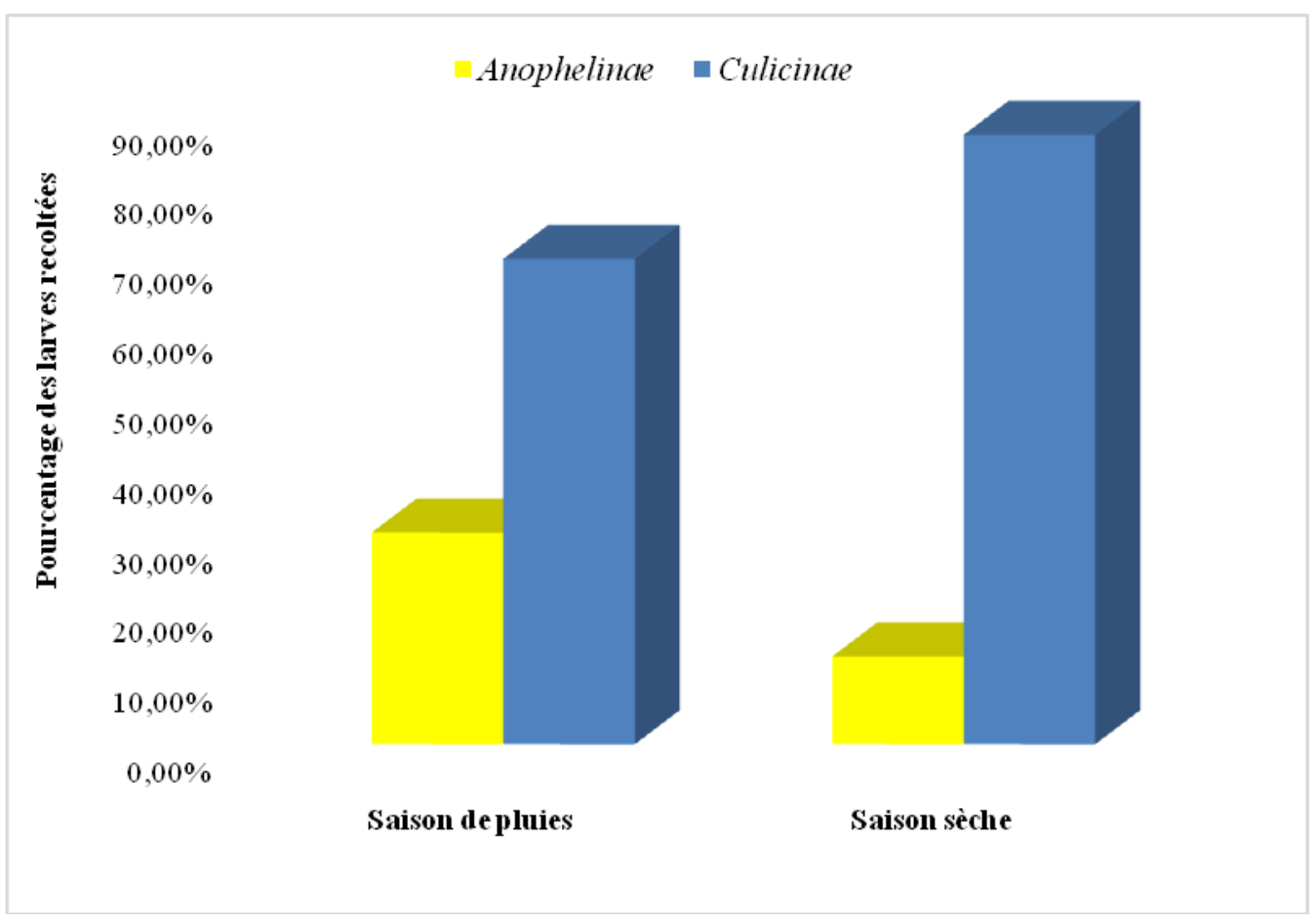

Figure 5 : Pourcentage des larves de moustiques récoltées par sous-famille et par saison. 
Tableau 2 : Production larvaire totale dans la région de Mouila par station prospectée.

\begin{tabular}{lll}
\hline Stations prospectées & Saison sèche & Saison pluvieuse \\
\cline { 2 - 3 } Camp Moutassou & $\mathbf{n}^{\mathbf{1}(\boldsymbol{\%})}$ & $\mathbf{n}^{\mathbf{2}(\boldsymbol{\%})}$ \\
Village Moutambe Sane Foumou & $1514(35,47)$ & $1393(17,71)$ \\
Camp Mboukou & $1162(27,22)$ & $2120(26,95)$ \\
Camp Doubou & $929(21,76)$ & $1016(12,91)$ \\
Village Mboukou & $0(0)$ & $57(0,72)$ \\
Village Doubou & $86(2,01)$ & $700(8,90)$ \\
Camp Ngounié & $310(7,26)$ & $33(0,42)$ \\
PK 19 & $183(4,29)$ & $1121(14,25)$ \\
Camp Mavassa & $63(1,48)$ & $575(7,31)$ \\
Total & $22(0,51)$ & $852(10,83)$ \\
\hline
\end{tabular}

$\mathbf{n}^{1}$ : Effectif total de larves récoltées en saison sèche, $\mathbf{n}^{2}$ : Effectif total de larves récoltées en saison pluvieuse.

Tableau 3 : Abondance larvaire dans les différentes stations prospectées à Mouila.

\begin{tabular}{lllcc}
\hline \multirow{2}{*}{ Stations prospectées } & \multicolumn{2}{c}{ Saison sèche } & \multicolumn{2}{c}{ Saison pluvieuse } \\
\cline { 2 - 5 } Camp Moutassou & \multicolumn{1}{c}{$\mathbf{n}^{\mathbf{a}(\%)}$} & \multicolumn{1}{c}{$\mathbf{n}^{\mathbf{b}}(\%)$} & $\mathbf{n}^{\mathbf{a}}(\%)$ & $\mathbf{n}^{\mathbf{b}}(\%)$ \\
Village Moutambe Sane Foumou & $89(14,83)$ & $1073(29,25)$ & $6(0,25)$ & $2114(38,59)$ \\
Camp Mboukou & $244(40,67)$ & $685(18,67)$ & $251(10,51)$ & $765(13,96)$ \\
Camp Doubou & $0(0)$ & $0(0)$ & $45(1,88)$ & $12(0,22)$ \\
Village Mboukou & $0(0)$ & $86(2,34)$ & $700(29,30)$ & $0(0)$ \\
Village Doubou & $0(0)$ & $310(8,45)$ & $0(0)$ & $33(0,60)$ \\
Camp Ngounié & $20(3,33)$ & $163(4,44)$ & $84(3,52)$ & $1037(18,93)$ \\
PK 19 & $63(10,5)$ & $0(0)$ & $6(0,25)$ & $569(10,39)$ \\
Camp Mavassa & $0(0)$ & $22(0,60)$ & $518(21,68)$ & $334(6,10)$ \\
\hline \multicolumn{1}{c}{$\mathbf{n}^{\mathbf{a}}:$ Effectif de larves d'Anophelinae récoltées, } & \\
\end{tabular}


Tableau 4 : Variation saisonnière des effectifs des moustiques adultes par saison.

\begin{tabular}{lll}
\hline Moustiques & Saison sèche & Saison pluvieuse \\
\hline Adultes d'Anophelinae & $240(20,76 \%)$ & $1510(29,19 \%)$ \\
Adultes de Culicinae & $916(79,24 \%)$ & $3663(70,81 \%)$ \\
Total & 1156 & 5173 \\
\hline
\end{tabular}

Tableau 5 : Variation saisonnière des effectifs des adultes en fonction des stations.

\begin{tabular}{|c|c|c|c|c|c|c|}
\hline \multirow{2}{*}{ Stations prospectées } & \multicolumn{2}{|c|}{ Saison sèche } & \multirow{2}{*}{$\begin{array}{l}\text { Total } 1 \\
\mathrm{n}(\%)\end{array}$} & \multicolumn{2}{|c|}{ Saison pluvieuse } & \multirow{2}{*}{$\begin{array}{l}\text { Total } 2 \\
\mathbf{n}(\%)\end{array}$} \\
\hline & $\mathrm{n}^{\mathrm{a}}(\%)$ & $\mathrm{n}^{\mathrm{b}}(\%)$ & & $\mathrm{n}^{\mathrm{a}}(\%)$ & $\mathrm{n}^{\mathrm{b}}(\%)$ & \\
\hline Camp Moutassou & $46(19,17)$ & $248(27,07)$ & $294(25,43)$ & $524(34,70)$ & $318(8,68)$ & $842(16,28)$ \\
\hline Village Moutambe Sane F. & $13(5,42)$ & $34(3,71)$ & $47(4,07)$ & $6(0,40)$ & $1696(46,30)$ & $\begin{array}{l}1702 \\
(32,90)\end{array}$ \\
\hline Camp Mboukou & $170(70,83)$ & $469(51,20)$ & $639(55,28)$ & $251(16,62)$ & $659(17,99)$ & $910(17,59)$ \\
\hline Camp Doubou & $0(0)$ & $47(5,13)$ & $47(4,07)$ & $29(1,92)$ & $12(0,33)$ & $41(0,79)$ \\
\hline Village Mboukou & $0(0)$ & $30(3,28)$ & $30(2,59)$ & $306(20,26)$ & $0(0)$ & $306(5,91)$ \\
\hline Village Doubou & $0(0)$ & $0(0)$ & $0(0)$ & $0(0)$ & $33(0,90)$ & $33(0,64)$ \\
\hline Camp Ngounié & $11(4,58)$ & $52(5,68)$ & $63(5,45)$ & $77(5,10)$ & $527(14,39)$ & $604(11,68)$ \\
\hline PK 19 & $0(0)$ & $29(3,17)$ & $29(2,51)$ & $6(0,40)$ & $220(6,01)$ & $226(4,37)$ \\
\hline Camp Mavassa & $0(0)$ & $7(0,76)$ & $7(0,60)$ & $311(20,60)$ & $198(5,40)$ & $509(9,84)$ \\
\hline Total & 240 & 916 & 1156 & 1510 & 3663 & 5173 \\
\hline
\end{tabular}

Tableau 6 : Répartition des gîtes positifs identifiés dans les stations prospectées.

\begin{tabular}{lccc}
\hline \multirow{2}{*}{ Stations prospectées } & Saison sèche & Saison pluvieuse & Total \\
\cline { 2 - 4 } & $\mathbf{n}(\%)$ & $\mathbf{n}(\%)$ & $\mathbf{n}(\%)$ \\
\hline Camp Moutassou & $13(32,5)$ & $20(25,97)$ & $33(28,20)$ \\
Village Moutambe Sane Foumou & $8(20)$ & $4(5,19)$ & $12(10,26)$ \\
Camp Mboukou & $5(12,5)$ & $7(9,09)$ & $12(10,26)$ \\
Camp Doubou & $5(12,5)$ & $2(2,60)$ & $7(5,98)$ \\
Village Mboukou & $2(5)$ & $13(16,88)$ & $15(12,82)$ \\
Village Doubou & $0(0)$ & $6(7,79)$ & $6(5,13)$ \\
Camp Ngounié & $4(10)$ & $8(10,39)$ & $12(10,26)$ \\
PK 19 & $2(5)$ & $7(9,09)$ & $9(7,69)$ \\
Camp Mavassa & $1(2,5)$ & $10(12,99)$ & $11(9,40)$ \\
\hline
\end{tabular}

n: nombre de gîtes larvaires positifs recensés, \% : pourcentage de gîtes larvaires positifs identifiés. 


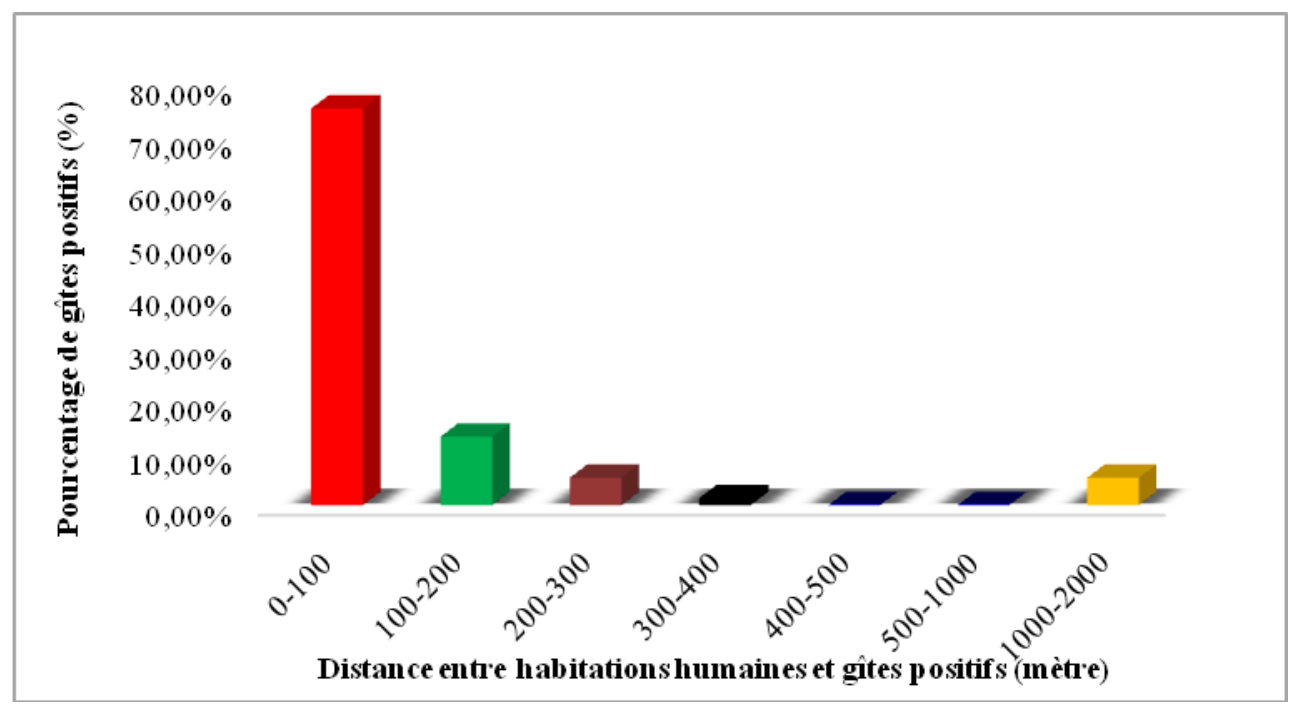

Figure 6 : Proximité entre les gîtes positifs et les maisons en saison de pluies à Mouila.

\section{DISCUSSION}

Les prospections larvaires réalisées dans ce travail ont montré que la zone d'étude présente une diversité de gîtes larvaires. La majorité de ces gîtes larvaires a été créé par l'homme du fait de ses activités (activités agricoles, surfaces d'eau artificielles, etc.). Ces gîtes, notamment ceux ayant au moins une larvese trouvent dans l'environnement immédiat despopulations humaines (moins de $400 \mathrm{~m}$ des habitations). De plus, ces gîtes sont propices au développement des moustiques des genres Culex, Aedes et Anopheles qui sont des vecteurs majeurs de nombreux pathogènes responsables de nombreuses pathologies dont le paludisme, le chikungunya, les filarioses, la dengue, la fièvre jaune, le virus zika (Rodhain, 2015). Ces résultats sont similaires à ceux de Tia et al. (2016) qui ont montré la responsabilité des habitants dans la mise en place des conditions propices au développement et au maintien des moustiques via la création de leurs habitats larvaires. Aussi, la proximité des sites de développement des moustiques avec les habitations pourrait constituer un risque de santé et de nuisance pour les populations humaines environnantes tel qu'observés par Dambach et al. (2009) au Burkina Faso, Koné et al. (2013) et Tia et al. (2016) en Côte
d'Ivoire. La présence de ces gîtes larvaires pourrait augmenter les risques de transmission d'autres agents infectieux par ces moustiques. Par exemple, les cas de paludisme et d'arboviroses peuvent s'accroître dans la population locale quand ces gîtes sont favorables au développement des stades larvaires des populations d'Anopheles, Culex et Aedes, notamment en saison des pluies (Grard et al., 2007 ; Sy et al., 2016).

$\mathrm{Au}$ cours de cette étude, la production larvaire culicidienne a été plus élevée en saison pluvieuse qu'en saison sèche. Cette abondance est liée probablement, d'une part, à la forte quantité des gîteslarvaires permanentsprésents dans la zone d'étude et, d'autre part, au fait que la saison pluvieuse offre des possibilités de choix des gîtes aux femelles des différentes espèces de moustiques aussi bien en qualité qu'en quantité. Or, cela n'est pas le cas pour la saison sèche où certains gîtes favorables au développement des moustiques s'assèchent ou disparaissent ; cela a pour conséquence une rareté des gites larvaires.

Cependant, la faible proportion des gîtes positifs identifiés en saison des pluies, serait probablement liée aux fortes précipitationsobservées à Mouila au cours de notre étude. En effet, ces pluies auraient 
lessivécertains gîtes larvaires qui seraient à priori favorables audéveloppement des moustiques. De même, le pourcentage des gîtes permanents a été moins élevé en saison des pluies qu'en saison sèche. Cette différence serait due au fait que lors de la saison pluvieuse, les différents gîtes permanents recensés en saison sèche ont été détruits par l'homme pour ses activités, dont la construction des routes, des habitations, etc.

Deux sous-familles de moustiques, à savoir les Anophelinae et les Culicinae, ont été identifiées avec une prédominance des larves de Culicinae. Cette abondance des individus de Culicinae pourrait s'expliquer par le niveau de la nature des gîtes larvaireset par la capacité de résilience des larves de Culicinae (Korba et al., 2016). Ces observations corroborent celles faites par Korba et al. (2016) en Algérie qui ont montré que les espèces de moustiques appartenant aux Culicinae présentent des fortes capacités d'adaptation leur permettant de se développer dans plusieurs types d'habitats.Des résultats identiques ont été obtenus par Saotoing et al. (2011) qui ont relevé que les stades immatures du genre Culexont une grande élasticité écologique et sont capables de coloniser différents milieux. D'ailleurs, les gîtes larvaires colonisés au cours de cette étude par les moustiques du genre Culexétaient variés (flaques d'eau, étangs, mares, récipients de stockage d'eau (seau, fût), retenues d'eau, fosses). Ces gîtes larvaires étaient pour la plupart caractérisés par la présence des papiers, les bouteilles, les débris végétaux, etc.Selon, Alami et al. (2010), la pullulation des espèces de moustiques est conditionnée par la nature du gîte larvaire. Pour ces auteurs, la nature du gîte favorise l'une ou d'autres espèces culicidiennes selon les caractéristiques du gîte : stagnant ou courant, pollué ou non, dépourvu ou riche en végétation.Par contre, la faible quantité des larves d'Anopheles gambiae s.l. (Anophelinae) semble être est liée à leur bio écologie et au fait que les gîtes larvaires identifiés dans la zone d'étude ne présentent pas toutes les conditions requises pour leur développement.Selon les travaux conduits par
Betsi et al. (2012) et Sy et al. (2016), les femelles d'Anopheles gambiae s.l. préfèrent pondre leurs œufs dans les collections d'eau ensoleillées et dépourvues de végétation. Ces femellesprésentent aussi un tropisme positif pour les eaux claires douces et les eaux saumâtres pour le dépôt de leurs œufs. Dans cette étude, les collections d'eau ensoleillées (trous dans le sol, retenues d'eau et empreintes de pneus) et dépourvues de végétation n'étaient peu abondantes. De plus, les gîtes d'An. Gambiae s.l. rencontrés dans les sites d'étude sont des gîtes à $\mathrm{pH}$ soit acide ou neutre et à température relativement basse par rapport à celle des gîtes d'Aedes sp. Et Culex sp.

De toutes les stations prospectées, ce sont les camps Moutassou, Mboukou et Ngounié, et le village Moutambe Sane Foumou qui ont été les plus productifs en larves de moustiques. L'abondance de ces larves dans ces stations serait liée aux activités agricoles (palmeraies, cultures) pratiquées. Ces activités humaines ont créé ainsi des conditions propices à leur présence. Aussi, la proximité de ces stations avec les habitations pourrait constituer un risque majeur dans la transmission des maladies vectorielles (Dambach et al., 2009 ; Ahmad et al., 2011). De plus, le maximum des moustiques adultes a été enregistréau cours de la saison pluvieuse et dans les stations susmentionnées.

\section{Conclusion}

Les résultats obtenus au cours de cette étude révèlent que les moustiques se développent dans tous les types de points d'eau mais préfèrent les surfaces d'eau artificielles. La majorité des habitats larvaires positifs se trouvait dans l'environnement immédiat des populations humaines. Le maximum de gîtes larvaires et de larves a été enregistré en saison pluvieuse avec une abondance des larves de la sous-famille des Culicinae comparativement à celles de la sous-famille des Anophelinae. Cette production larvaire est tributaire de la saison, des pratiques humaines et de la durée de conservation de l'eau dans les contenants artificiels. Parmi les 9 stations prospectées, les 
stations les plus fructueuses en larves étaient le village Moutambe Sane Foumou, les camps Moutassou, Mboukou et Ngounié ont été les zones où le maximum de larves a été obtenu.

$\mathrm{Au}$ regard de nos résultats, la pullulation de ces moustiques vecteurs d'agents de maladies serait liée à l'insalubrité de l'environnement immédiatet aux activités anthropiques qui créent et assurent le maintien des sites de reproduction des moustiques. Aussi, il est essentiel de mener une étude entomologique et parasitologique dans cette région afin d'évaluer le niveau de transmission du paludisme et les risques de transmission des arbovirus au sein de la population locale.

\section{REMERCIEMENTS}

Ce travail a été réalisé grâce à l'appui financier, institutionnel et logistique du Laboratoire d'Ecologie Vectorielle (LEV), de l'Université d'Abomey-Calavi (UAC), de l'Institut Régional de Santé Publique (IRSP), de l'Institut de Recherche en Ecologie Tropicale (IRET), du Programme National de Lutte contre le Paludisme (PNLP), de l'Université des Sciences et Techniques de Masuku (USTM) et de la société Olam Palm Gabon (OPG). Nos sincères remerciements à l'OMS-Gabon pour son aide matérielle.

\section{CONFLIT D'INTERETS}

Les auteurs déclarent qu'il n'existe aucun conflit d'intérêts lié à la présente étude.

\section{CONTRIBUTIONS DES AUTEURS}

AAK et CRZK ont participé à la collecte des données sur le terrain et ont pris une part active à l'organisation, au traitement des données, à la rédaction et à la correction de toutes les parties du manuscrit. RMN et POO ont participé à la collecte des données sur le terrain et à la relecture du manuscrit. GKK, LSD et JFM sont les initiateurs du projet scientifique de ce manuscrit. Ils ont assuré la conception et l'administration du projet. Ils ont supervisé toutes les activités du projet sur le terrain et au laboratoire. Ils se sont aussi impliqués dans les travaux de terrain et de laboratoire en participant à la collecte des larves et nymphes des moustiques et à leur élevage au laboratoire. PC, BM et PAN ont participé à la conception du projet, à la relecture et à la correction du manuscrit. Tous les auteurs remercient les relecteurs qui ont participé à l'amélioration de la qualité de cet article.

\section{REFERENCES}

Abagli AZ, Alavo TBC, Brodeur J. 2014. Microorganismes entomopathogènes, prédateurs et parasites des moustiques: Perspectives pour la lutte raisonnée contre les vecteurs du paludisme en Afrique subsaharienne. International Journal of Biological and Chemical Sciences, 8(1): 340-354.

DOI: http://dx.doi.org/10.4314/ijbcs.v8i1.29

Ahmad R, Ali WNM, Nor ZM, Ismail Z, Hadi AA, Ibrahim MN, Lim LH. 2011. Mapping of mosquito breeding sites in malaria endemic areas in Pos Lenjang, Kuala Lipis, Pahang, Malaysia. Malaria Journal, 10: 361-372. DOI: 10.1186/14752875-10-361.

Akono NP, Mbouangoro A, Mbida-Mbida A, Ndo C, Peka-Nsangou MF, Kekeunou S. 2017. Le complexe d'espèces Anopheles gambiae et le gène de résistance $\mathrm{Kdr}$ en périphérie de Douala, Cameroun. Bulletin de la Société de Pathologie Exotique, 110 : 122-129.

Alami EOA, El Hilali O, Benlamlih M, Merzouki M, Raiss N, Ibensoudakouraichi S, Himmi O. 2010. Etude entomologique, physico-chimique et bactériologique des gîtes larvaires de localités à risque potentiel pour le paludisme dans la Ville de Fès. Bulletin de l'Institut Scientifique, Rabat, 32(2): 119 127.

Badolo A, Ilboudo-Sanogo E, Sanon A, Ouédraogo AP. 2012. Evaluation de la protection personnelle contre les Anophelinae par utilisation de moustiquaires détériorées imprégnées de répulsifs. International Journal of Biological and Chemical Sciences, 6(1): 237-247.

DOI: http://dx.doi.org/10.4314/ijbcs.v6i1.20

Baldacchino F, Paupy C. 2010. Clé de détermination des Culicidae présents en Afrique Centrale et au Gabon. Document de travail. IRD/CIRMF.p. 108. 
Becker N, Petric D, Zgomba M, Boase C, Madon M, Dahl C, Kaiser A. 2010. Mosquitoes and Their Control. Second Edition Springer, Edition 2010. p. 87. DOI : 10.1007/978-3-540-92874-4

Betsi AN, Tchicaya ES, Koudou BG. 2012. Forte prolifération de larves d'An. gambiae et An. funestus en milieux rizicoles irrigués et non irrigués dans la région forestière ouest de la Côte-d'Ivoire. Bulletin de la Société de Pathologie Exotique, 105: 220-229. DOI : 10.1007/s13149-012-0219-z

Coffinet T, Rogier C, Pages F. 2009. Evaluation de l'agressivité des anophèles et du risque de transmission du paludisme, méthodes utilisées dans les armées françaises. Medecine Tropicale, 69: 109122.

Dambach P, Sie A, Lacaux JP, Vignolles C, Machault V, Sauerborn R. 2009. Using high spatial resolution remote sensing for risk mapping of malaria occurrence in the Nouna district, Burkina Faso. Global Health Action, 2(0): 1-7. DOI: 10.3402/gha.v2i0.2094

Diedhiou SM, Niang EHA, Doucoure S, Samb B, Konate A, Cissokho S, Ndiaye A, Wotodjo AN, Chauvancy G, Gadiaga L, Dotson E, Thwing J, Konate L, Sokhna C, Faye O. 2016. Distribution and characterization of anopheline larval habitats in flooded areas of the Dakar suburbs. Journal of Parasitology and Vector Biology, 8(7): 61-73. DOI : 10.5897/JPVB2016.241

Ecosphère. 2011. Etude d'Impact Environnemental relative à l'implantation d'une palmeraie dans la zone de Mouila (Lot 1). Rapport d'EIES. Olam Palm Gabon.p. 300.

Ecosphère. 2014. Pré-évaluation de la zone du projet d'aménagement d'une palmeraie à Mouila Lot 3. Rapport de mission. Olam Palm Gabon.p. 30.

Elissa N, Migot NF, Luty A, Renaut A, Touré F, Vaillant M, Lawoko M, Yangari P, Mayombo J, Lekoulou F, Tshipamba P, Moukagni R, Millet P, Deloron P. 2003. Relationship between entomological inoculation rate, Plasmodium falciparum prevalence rate, and incidence of malaria attack in rural Gabon. Acta Tropica, 85: 355-361.

Grard G, Caron M, Mombo I, Nkoghe D, Mboui-Ondo S, Jiolle D, Fontenille D, Paupy C, Leroy EM. 2014. Zika Virus in
Gabon (Central Africa)-2007: A new threat from Aedes albopictus? PLoS Neglected Tropical Diseases, 8(2): 1-6. e2681. DOI:10.1371

Ikram M, Chibani A, Alemad A, Alkhali A, Belala A, Hadji M, Belghyti D, El Kharrim K. 2016. Etude écologique et entomologique des gîtes larvaires des Culicidés de la Province de Kenitra (Maroc). European Scientific Journal, 12(32): 1857- 7431. DOI : 10.19044/esj.2016.v12n32p398

Kenawy MA, Ammar SE, Abdel-Rahman HA. 2013. Physico-chemical characteristics of the mosquito breeding water in two urban areas of Cairo Governorate, Egypt. Journal of Entomological and Acarological Research, 45(17): 96-100. DOI : 10.4081/jear.2013.e17

Koné AB, Konan YL, Coulibaly ZI, Fofana D, Guindo-Coulibaly N, Diallo M, Doannio JMC, Ekra KD, Odehouri-Koudou P. 2013. Evaluation entomologique du risque d'épidémie urbaine de fièvre jaune survenue en 2008 dans le district d'Abidjan, Côte d'Ivoire. Médecine et Santé Tropicales, 23 : 66-71. DOI : 10.1684/mst.2013.0153

Korba RA, Alayat MS, Bouiba L, Boudrissa A, Bouslama Z, Boukraa S, Francis F, Failloux AB, Boubidi SC. 2016. Ecological differentiation of members of the Culex pipiens complex, potential vectors of West Nile virus and Rift Valley fever virus in Algeria. Parasites \& Vectors, 9: 455-465. DOI : 10.1186/s13071-016-1725-9

Koudou BG, Tano Y, Doumbia M, Nsanzabana C, Cissé G, Girardin O, Dao D, N'goran EK, Vounatsou P, Bordmann G, Keiser J, Tanner M, Utzinger J. 2005. Malaria transmission dynamics in central Côte d'Ivoire: the influence of changing patterns of irrigated rice agriculture. Medical and Veterinary Entomology, 19(1): 27-37. DOI : 10.1186/1756-3305-391

Koumba AA, Zinga-Koumba CR, MintsaNguema R, Bi Zahouli JZ, Ovono AM, Souza A, Ketoh GK, Djogbenou LS, M'batchi B, Mavoungou JF. 2018a. Preliminary evaluation of the insecticide susceptibility in the culicid fauna, particularly malaria plasmodium and arbovirus vectors in the region of Mouila, South-west Gabon. Indian Journal of 
Medical Research and Pharmaceutical Sciences, 5(4): 105-117. DOI: 10.5281/zenodo. 1236958

Koumba AA, Zinga-Koumba CR, MintsaNguema R, Sevidzem SL, Djogbenou LS, Akono PN, Ketoh GK, Faye O, M'batchi $B$ and Mavoungou JF. 2018b. Identification of the knockdown resistance (Kdr) mutations in Anopheles gambiae s.l. in the Mouila area, South west Gabon. Journal of Entomology and Zoology Studies, 6(3): 602-607.

Makanga BK. 2016. Ecologie de la transmission des Plasmodium simiens au Gabon. Thèse de $3^{\text {ème }}$ cycle, Ecole Doctorale Régionale de Franceville, Franceville, p. 191.

Mounioko F, Dibakou ES, Zinga-Koumba CR, Mbang-Nguema OA, Acapovi-Yao G, Shango M, Mavoungou JF. 2015. Rythme d'activité journalière de Glossina fuscipes fuscipes, vecteur majeur de la trypanosomiase humaine africaine dans le parc national de Moukalaba Doudou (Sud-Ouest Gabon). International Journal of Biological and Chemical Sciences, 9(1): 419-429. DOI: http://dx.doi.org/10.4314/ijbcs.v9i1.35

Mounioko F, Zinga-Koumba CR, Koumba AA, Maganga GD, Tamesse JL, Simo G, M'batchi B, Mavoungou JF. 2018. Contribution to the evaluation of the diversity and abundance of hematophage dipteries in the Moukalaba Doudou National Park (southern Gabon) in rainy season. International Journal of Advanced Research, 6(2): 1170-1182. DOI: 10.21474/IJAR01/6498

Mourou JR, Coffinet T, Jarjaval F, Cotteaux C, Pradines E, Godefroy L, Kombila M, Pagès F. 2012. Malaria transmission in Libreville: results of a one year survey. Malaria Journal, 11: 1-14. DOI : 10.1186/1475-2875-11-40

Ndjimbi F. 2013. Les populations Gabonaises face à l'insécurisassions foncière, étude d'impact des plantations agro-industrielles de palmiers à huile et d'hévéa sur les populations du Gabon. ONG Brainforest, p. 72.

Paupy C, Makanga B, Ollomo B, Rahola N, Durand P, Magnus J, Willaume E, Renaud F, Fontenille D, Prugnolle F. 2013. Anopheles moucheti and Anopheles vinckei are candidate vectors of Ape Plasmodium Parasites, Including Plasmodium praefalciparum in Gabon.
PLoS ONE, 8(2): 1-6. DOI : 10.1371/journal.pone.0057294

PNLP. 2010. Statistique épidémiologique du Gabon. Rapport annuel d'activités. Ministère de la Santé, Libreville, p. 30.

Rodhain F. 2015. Le parasite, le moustique, l'homme et les autres: Essai sur l'épidémiologie des maladies à vecteurs, Edition Dorcas, p. 440.

Saotoing P, Njan-Nloga AM, TchuenguemFohouo FN, Yaya O, Messi J. 2011. Bioécologie des larves de Culicidae (Diptera) dans la ville de Maroua, Extreme-Nord du Cameroun. Sciences et Médecines en Afrique, 3(1): 417-422.

Serandour J. 2007. Contribution à l'étude des moustiques anthropophiles de France: le cas particulier du genre Coquillettidia. Thèse de doctorat de $3^{\text {ème }}$ Cycle, Université Joseph Fournier-Grenoble I, Grenoble, p. 213.

Sy O, Konaté L, Ndiaye A, Dia I, Diallo A, Taïrou F, Bâ EL, Gomis JF, Ndiaye JL, Cissé B, Gaye O, Faye O. 2016. Identification des gîtes larvaires d'anophèles dans les foyers résiduels de faible transmission du paludisme « hotspots » au centre-ouest du Sénégal. Bulletin de la Société de Pathologie Exotique, 109: 31-38. DOI : 10.1007/s13149-016-0469-2

Talipouo A, Ntonga-Akono P, Tagne D, Mbida-Mbida A, Etang J, TchoffoFobasso R, Ekoko W, Binyang J, Dongmo A. 2017. Comparative study of Culicidae biodiversity of Manoka island and Youpwe mainland area, Littoral, Cameroon. International Journal of Biosciences, $10 \quad$ (4): 9-18. DOI : http://dx.doi.org/10.12692/ijb/10.4.9-18

Tia E, Gbalegba NGC, M'bra KR, Kaba A, Boby OAM, Koné M, Chouaibou M, Koné B, Koudou GB. 2016. Etude du niveau de production larvaire d'Anopheles gambiae s.l. (Diptera : Culicidae) dans les différents types de gîtes à Oussou-yaokro au Centre-Ouest et à Korhogo, au Nord (Côte d'Ivoire). Journal of Applied Biosciences, 105 : 10170-10182. DOI : http://dx.doi.org/10.4314/jab.v105i1.13. 